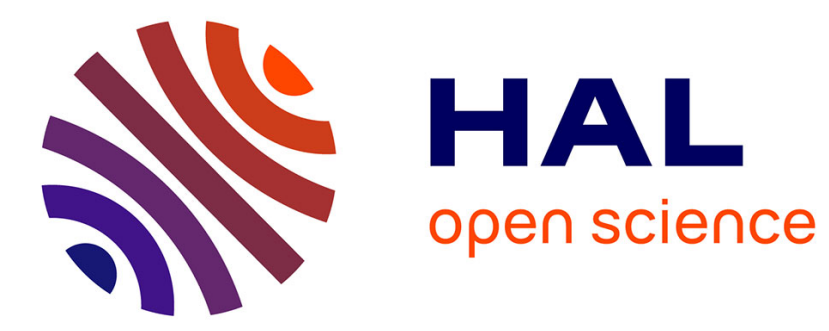

\title{
Unifying Suspension and Granular Rheology
}

François Boyer, Elisabeth Guazzelli, Olivier Pouliquen

\section{To cite this version:}

François Boyer, Elisabeth Guazzelli, Olivier Pouliquen. Unifying Suspension and Granular Rheology.

Physical Review Letters, 2011, 107, 10.1103/PhysRevLett.107.188301 . hal-01432411

\section{HAL Id: hal-01432411 \\ https://hal.science/hal-01432411}

Submitted on 11 Jan 2017

HAL is a multi-disciplinary open access archive for the deposit and dissemination of scientific research documents, whether they are published or not. The documents may come from teaching and research institutions in France or abroad, or from public or private research centers.
L'archive ouverte pluridisciplinaire HAL, est destinée au dépôt et à la diffusion de documents scientifiques de niveau recherche, publiés ou non, émanant des établissements d'enseignement et de recherche français ou étrangers, des laboratoires publics ou privés. 


\title{
Unifying Suspension and Granular Rheology
}

\author{
François Boyer, ${ }^{*}$ Élisabeth Guazzelli, and Olivier Pouliquen \\ IUSTI, Aix-Marseille Université, CNRS (UMR 6595) 5 rue E. Fermi, 13453 Marseille cedex 13, France
}

(Received 29 July 2011; published 24 October 2011)

\begin{abstract}
Using an original pressure-imposed shear cell, we study the rheology of dense suspensions. We show that they exhibit a viscoplastic behavior similarly to granular media successfully described by a frictional rheology and fully characterized by the evolution of the friction coefficient $\mu$ and the volume fraction $\phi$ with a dimensionless viscous number $I_{v}$. Dense suspension and granular media are thus unified under a common framework. These results are shown to be compatible with classical empirical models of suspension rheology and provide a clear determination of constitutive laws close to the jamming transition.
\end{abstract}

DOI: 10.1103/PhysRevLett.107.188301

PACS numbers: 47.57.Gc, $83.80 . \mathrm{Hj}$

The rheology of dispersions of solid particles in a fluid has been extensively studied since the theoretical derivation of an effective viscosity in the dilute regime undertaken by Einstein in 1905 [1]. Despite their relevance to practical applications, even the simplest case of nonBrownian hard spheres suspended in a Newtonian liquid remains poorly understood, especially in the concentrated regime [2]. Such mobile particulate systems are known to undergo a jamming transition exhibiting a divergence of their viscosity when the particle volume fraction reaches a maximum value $[3,4]$. A detailed characterization of this divergence is still missing and more generally the dense regime, where both hydrodynamic and contact interactions contribute to the suspension mechanics, lacks a unified view.

In this Letter, we provide a new perspective on the suspension rheology. Using an original experimental setup, we analyze the results within the theoretical framework that has recently led to express universal constitutive laws for dense granular flows [5-8]. A continuous description of flowing granular media has long remained a challenge [9] but a major step was achieved by considering pressureimposed flows; see Fig. 1(a): when an assembly of hard spheres (having diameter $d$ and density $\rho_{p}$ ) is sheared at a given shear rate $\dot{\gamma}$ under a confining pressure $P^{p}$, while letting the medium free to dilate or to compact, there is only one dimensionless control parameter $I=d \sqrt{\rho_{p} / P^{p}} \dot{\gamma}$ [6]. This inertial number I can be seen as the ratio between the inertial time of rearrangement $t_{\text {micro }}=d \sqrt{\rho_{p} / P^{p}}$ and the time of strain $t_{\text {macro }}=1 / \dot{\gamma}$. The granular rheology is completely determined by two functions of the inertial number: a friction law for the shear stress $\tau=\mu(I) P^{p}$ and a volume-fraction law $\phi(I)$ [5]. These scaling laws have proved very successful for describing dense granular flows in a wide range of flow configurations [8].

This granular paradigm can be applied to the case of particles suspended in a fluid of viscosity $\eta_{f}$ sheared at constant particle pressure $P^{p}$. Figure 1(b) depicts how $P^{p}$ can be imposed through a porous plate. If the Stokes number $S t=\rho_{p} d^{2} \dot{\gamma} / \eta_{f}$ is small, viscous forces are dominant at the particle scale and the internal time is now given by a viscous scaling $t_{\text {micro }}=\eta_{f} / P^{p}$. The system is no longer governed by the inertial number and a dimensionless viscous number should be used instead [10]

$$
I_{v}=\frac{\eta_{f} \dot{\gamma}}{P^{p}} .
$$

Following the approach used in the dry-granular case, constitutive laws can be expressed as two functions of $I_{v}$

$$
\tau=\mu\left(I_{v}\right) P^{p} \quad \text { and } \quad \phi=\phi\left(I_{v}\right) .
$$

Only a few measurements of the effective friction $\mu$ have been performed, either indirectly [10] or in the quasistatic limit [11-13], i.e., at vanishing $I_{v}$. They suggest that the medium whether dry or immersed in a liquid exhibits a similar friction coefficient $\mu_{1} \sim 0.3$ when $I_{v} \rightarrow 0$. No determination of the volume-fraction law $\phi\left(I_{v}\right)$ has been reported so far. Therefore, a comprehensive description of the suspension rheophysics is lacking. The objective of the present work is to provide these two constitutive laws over a wide range of $I_{v}$.

Toward this goal, we have designed an original annular shear cell in which pressure-imposed measurements are performed as shown in Fig. 1(c). The suspension is confined between a static truncated cone and a rotating annulus having an internal (external) radius $R_{1}=44 \mathrm{~mm}\left(R_{2}=\right.$ $90 \mathrm{~mm}$ ). The choice of a conical bottom plate ensures that the shear rate is quasihomogeneous in the vertical and radial directions. In the initial position $(\delta=0$, see below), the suspension height $h(r)$ varies linearly from $H_{1}=$ $8.8 \mathrm{~mm}$ to $H_{2}=18 \mathrm{~mm}$, and the shear cell volume is $V_{0}=$ $255 \mathrm{~cm}^{3}$. Both the bottom and top plates are made bumpy by gluing $0.5 \mathrm{~mm}$ steel bars every $5 \mathrm{~mm}$. Two combinations of particles and fluid are used in the experiments: (i) poly(methyl methacrylate) (PMMA) spheres having diameter $d=1.10 \pm 0.05 \mathrm{~mm}$ in a Triton X-100/water/ zinc chloride mixture of viscosity $\eta_{f}=3.1 \mathrm{~Pa} \cdot \mathrm{s}$; 

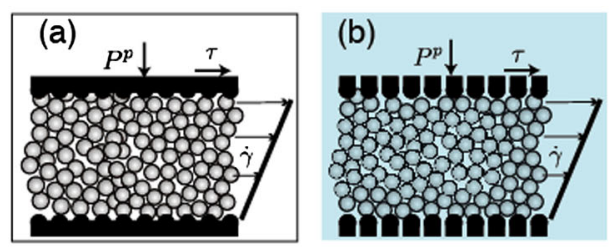

(c)

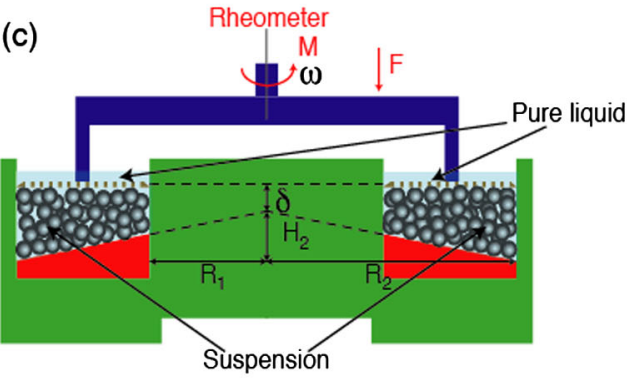

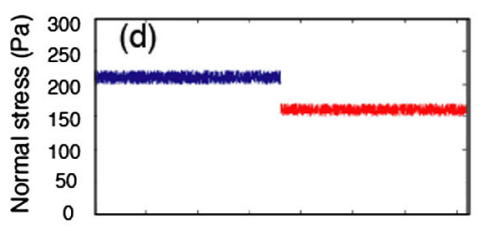
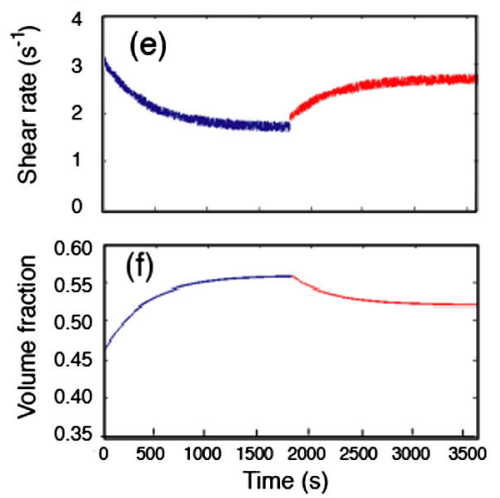

FIG. 1 (color online). Paradigmatic configurations of pressure-imposed shear of dry (a) or immersed (b) granular media; (c) experimental setup; Variation of shear rate (e) and volume fraction (f) in response to a change in applied normal stress (d), the shear stress being fixed (here, $\tau=150 \mathrm{~Pa}$ ).

(ii) polystyrene (PS) spheres $(d=0.58 \pm 0.01 \mathrm{~mm})$ in polyethylene glycol-ran-propylene glycol monobutylether $\left(\eta_{f}=2.15 \mathrm{~Pa} \cdot \mathrm{s}\right)$. In both systems, the density of the carrier fluid is closely matched to that of the suspended particles so that sedimentation can be neglected. The large size of the particles ensures that both colloidal forces and Brownian motion are negligible.

The originality of the experimental system is twofold. First, the interstitial fluid can pass through the top plate which is porous having $5 \mathrm{~mm}$ holes covered by a $200 \mu \mathrm{m}$ Nylon mesh. Second, the normal force $F$ applied to the top plate is imposed (but not its vertical position). The volume fraction is thus not fixed, but adjusts to the imposed shear. The experimental procedure is the following. The cell is filled with the desired amount of fluid and particles. The porous top plate is then lowered to the initial position for which $\delta=0$, see Fig. 1(c). The amount of particles is chosen such that the volume fraction at this initial stage is equal to $\phi_{0}=0.565$ (for most of the experiments). Changing $\phi_{0}$ does not significantly affect the results as seen by the good collapse of the data seen in Fig. 2. This suggests the absence of shear-banding effect. The top plate is driven by a MCR 501 (Anton Paar) rheometer at a given torque $M$ and normal force $F$, and the rotational speed $\omega$ and relative position $\delta$ are measured. This leads to the determination of the shear stress $\tau=3 M / 2 \pi\left(R_{2}^{3}-R_{1}^{3}\right)$, the granular pressure $P^{p}=\tilde{F} / \pi\left(R_{2}^{2}-R_{1}^{2}\right)[\tilde{F}$ being the normal force $F$ compensated for the top plate buoyancy], the mean shear rate averaged across the annulus $\dot{\gamma}=$ $\langle\omega r / h(r)\rangle$, and the particle volume fraction $\phi=$ $\phi_{0} /[1+f(\delta)]$ with $f(\delta)=\left[3 R_{2}\left(R_{2}^{2}-R_{1}^{2}\right) \delta\right] /\left[2\left(R_{2}^{3}-\right.\right.$ $\left.R_{1}^{3}\right) H_{2}$ ]. After a transient shown in Figs. 1(d)-1(f), where both $\phi$ and $\dot{\gamma}$ evolves in time, the system eventually reaches a steady state (in typically $30 \mathrm{~min}$ which corresponds to the slow vertical displacement of the top plate in the viscous fluid). In this steady state, the applied normal force $\tilde{F}$ is only balanced by the shear-induced granular pressure $P^{p}$ exerted by the particles on the top plate and this experiment then mimics the configuration of Fig. 1(b). Note that no hysteresis is observed and that the long time records show no slow variation. This suggests that particle migration effects are negligible in the present system.

The flow curves $\tau(\dot{\gamma})$ obtained at different applied pressures $P^{p}$ are shown in the inset of Fig. 2(a). When $P^{p}$ is imposed, the shear stress $\tau$ is an increasing function of $\dot{\gamma}$ and presents a yield stress $\tau_{y}$ at vanishing $\dot{\gamma}$. Increasing $P^{p}$ drastically shifts the flow curves to higher values. In order to test the relevance of the dimensional argument expressed by Eq. (1) and (2), the effective friction coefficient $\mu=\tau / P^{p}$ is plotted against the viscous number $I_{v}=$ $\eta_{f} \dot{\gamma} / P^{p}$ in Figs. 2(a) and 2(b). All the data obtained for different particle size, material, fluid viscosity, and initial number of particles collapse on a single curve $\mu\left(I_{v}\right)$. The friction coefficient $\mu$ tends to a finite value $\mu_{1}=\tau_{y} / P^{p}=$ $0.32 \pm 0.03$ at vanishing $I_{v}$ and increases with increasing $I_{v}$. The quasistatic value $\mu_{1}$ is similar to values obtained for dry-granular media. In contrast with the dry-granular rheology, $\mu\left(I_{v}\right)$ does not saturate at large $I_{v}$. As discussed below, this behavior is consistent with the additional viscous contribution to the total shear stress $\tau$.

The second constitutive law relative to the volume fraction Eq. (2) can also be tested. In Figs. 2(c) and 2(d), the particle volume fraction $\phi$ is plotted versus $I_{v}$ for the same experimental conditions. Again, the relevance of the viscous number $I_{v}$ is demonstrated by the collapse of all the data on a single curve $\phi\left(I_{v}\right)$. The volume fraction is a 

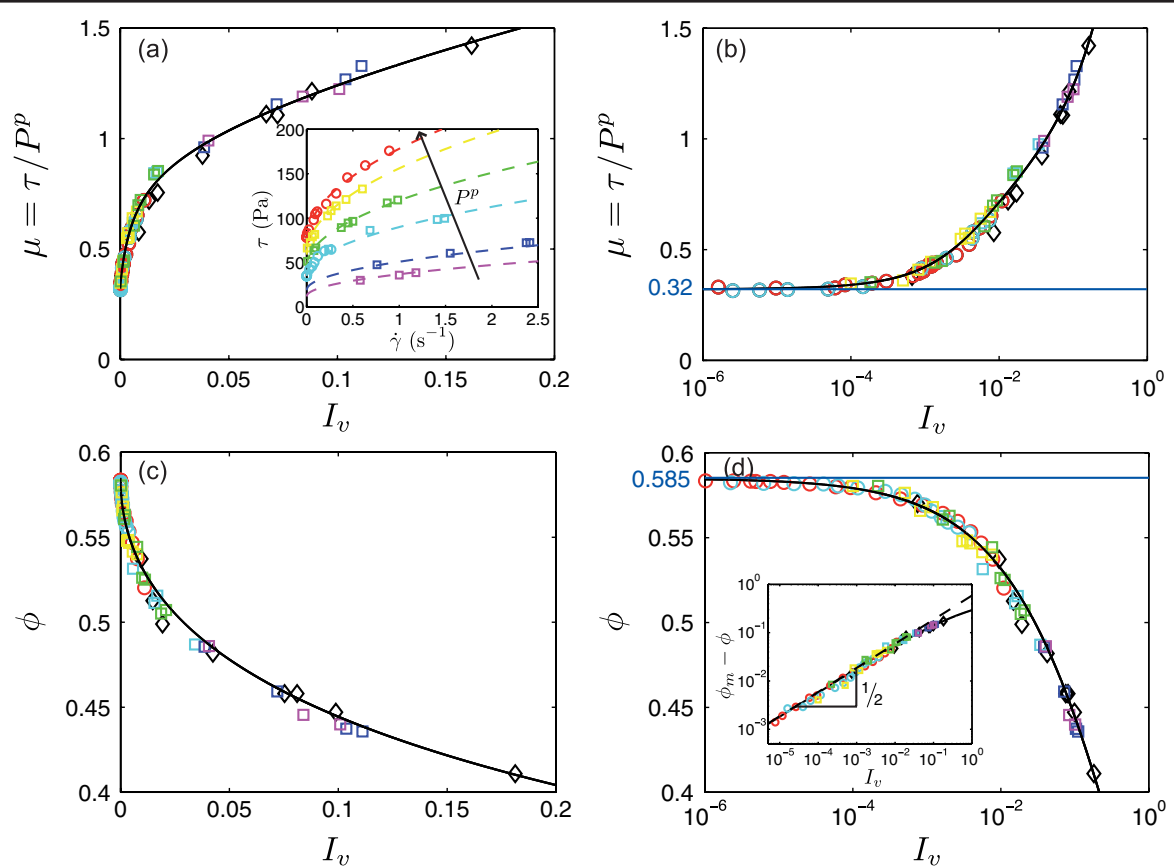

FIG. 2 (color online). Top: Friction coefficient $\mu=\tau / P^{p}$ as a function of the viscous number $I_{v}$ in linear (a) and semilogarithmic (b) scales; inset: flow curves at different pressures $P^{p}(35,53,109,142,193$, and $244 \mathrm{~Pa})$, for different particles [1.1 mm PMMA spheres $(\bigcirc), 0.58 \mathrm{~mm}$ PS spheres $(\square)]$ at $\phi_{0}=0.565$, and for the PS spheres at $\phi_{0}=0.433(\diamond)$. Bottom: Volume fraction $\phi$ as a function of $I_{v}$ in linear (c) and semilogarithmic (d) scales; inset: $\phi_{m}-\phi$ as a function of $I_{v}$. Solid lines are given by Eq. (6) and (7).

decreasing function of $I_{v}$ since the medium dilates when increasing the shear rate or diminishing the confining pressure $P^{p}$. From the semilogarithmic plot of $\phi\left(I_{v}\right)$ in Fig. 2(d), we can precisely determine the maximum volume fraction $\phi_{m}=0.585 \pm 0.002$ reached when $I_{v} \rightarrow 0$. Note that this value of the maximum packing fraction of an homogeneously sheared assembly of frictional spheres is close to the one reported in dry-granular media (the socalled critical volume fraction in critical state theory [14]) and significantly differs, as expected, from the randomclose packing fraction $\phi_{\text {rcp }} \simeq 0.635$. The inset of Fig. 2(d) shows that the asymptotic behavior of $\phi$ close to $\phi_{m}$ is given by a power-law $\left(\phi_{m}-\phi\right) \propto I_{v}^{1 / 2}$. This greatly differs from the behavior $\left(\phi_{m}-\phi\right) \propto I$ observed for drygranular media [8].

We have shown that the rheology of a dense suspension under imposed-pressure flow conditions is well described by the constitutive laws (2) and shares similar features with dry-granular rheology as long as the viscous number $I_{v}$ is substituted for the inertial number $I$. This frictional behavior of suspensions seems to differ from the classical view in terms of an effective viscosity [2]. However, these two formalisms can be reconciled as explained below. When a suspension is sheared at a constant volume fraction, shear and normal stresses scale viscously, i.e., $\propto \eta_{f} \dot{\gamma}$ and can be expressed as two functions of $\phi$

$$
\tau=\eta_{s}(\phi) \eta_{f} \dot{\gamma} \quad \text { and } \quad P^{p}=\eta_{n}(\phi) \eta_{f} \dot{\gamma},
$$

where $\eta_{s}(\phi)$ and $\eta_{n}(\phi)$ are the dimensionless effective shear and normal viscosities respectively $[2,15]$. Relating these constitutive laws to the above granular paradigm, Eq. (2), is straightforward. The volume fraction $\phi\left(I_{v}\right)$ being a monotonic function of the viscous number $I_{v}$, the inverse function $I_{v}(\phi)$ is defined unambiguously. Using Eq. (1) and the frictional constitutive law, both the particle pressure and the shear stress are found to scale viscously as

$$
P^{p}=\frac{1}{I_{v}(\phi)} \eta_{f} \dot{\gamma} \quad \text { and } \quad \tau=\frac{\mu\left[I_{v}(\phi)\right]}{I_{v}(\phi)} \eta_{f} \dot{\gamma} .
$$

Identifying these later equations with Eq. (3) provides

$$
\eta_{s}(\phi)=\frac{\mu\left[I_{v}(\phi)\right]}{I_{v}(\phi)} \quad \text { and } \quad \eta_{n}(\phi)=\frac{1}{I_{v}(\phi)} .
$$

The dimensionless effective shear and normal viscosities can then be computed from the data of Fig. 2 by plotting $\mu / I_{v}$ and $1 / I_{v}$ as functions of $\phi$, as shown in Fig. 3. Figure 3(a) shows that $\eta_{s}(\phi)=\mu / I_{v}$ increases with increasing $\phi$ and diverges when $\phi \rightarrow \phi_{m}$, as expected from the vanishing of $I_{v}$ when $\phi \rightarrow \phi_{m}$. Experimental data are in fairly good agreement with data found in the literature and, in particular, classical empirical correlations [2] such as those of Eilers (red dashed line) and Krieger \& Dougherty (green dashed line). It is important to stress that data have been obtained very close to $\phi_{m}$ (with values as close as $\phi_{m}-\phi \sim 5 \times 10^{-3}$ ) reaching huge values of $\eta_{s} \sim 10^{5}$. Figure 3(b) also shows similar increase and divergence of $\eta_{n}(\phi)=1 / I_{v}$ with $\phi$. These data are in good agreement with the scarce measurements 

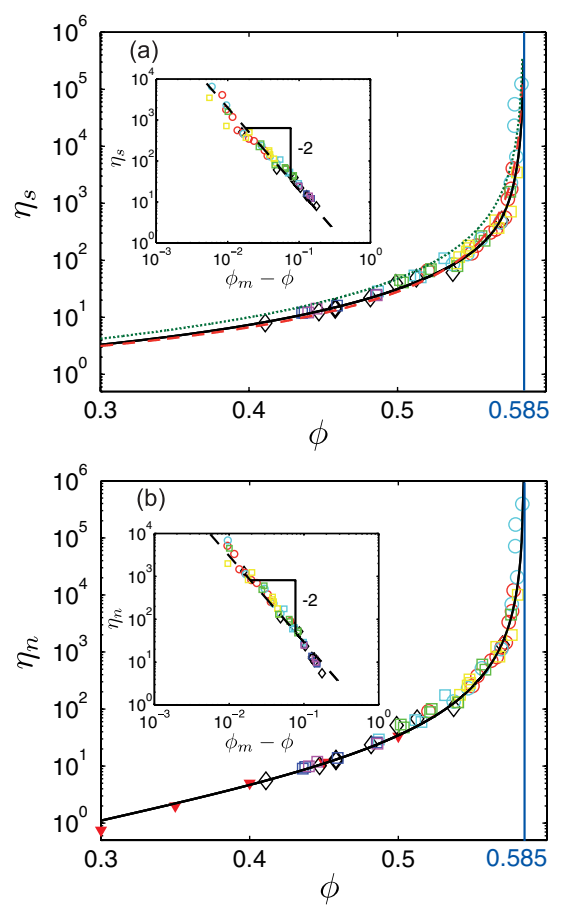

FIG. 3 (color online). Effective shear $\eta_{s}$ (a) and normal $\eta_{n}$ (b) viscosities versus $\phi$ (same symbols as in Fig. 2). (a) Empirical correlations of Eilers (red dashed line) and Krieger-Dougherty (green dashed line); see [2]; (b) particle-pressure measurements [16] (red down-triangles). Continuous lines are given by Eq. (8) and (9). Insets: logarithmic plots of $\eta_{s}$ and $\eta_{n}$ versus $\phi_{m}-\phi$.

of particle pressure [16] and greatly extends the range of volume fraction investigated.

The effective viscosities $\eta_{s}$ and $\eta_{n}$ present the same divergence $\left(\phi_{m}-\phi\right)^{-2}$ as stressed by the finite value of $\mu$ when $I_{v} \rightarrow 0$ and clearly evidenced in the insets of Fig. 3. It is worth noticing that these divergences are simply related to the behavior of $\phi$ at vanishing $I_{v}$ and can be directly inferred from the asymptotic form $\left(\phi_{m}-\phi\right) \propto I_{v}^{1 / 2}$.

We have shown that we can relate the frictional formalism of dense suspensions with their classical viscous rheology. We now propose constitutive laws which unify suspension and granular rheology as far as the friction law of dense suspensions $\mu\left(I_{v}\right)$ is modeled as the sum of two contributions, coming, respectively, from contact and hydrodynamic stresses

$$
\mu\left(I_{v}\right)=\underbrace{\mu_{1}+\frac{\mu_{2}-\mu_{1}}{1+I_{0} / I_{v}}}_{\mu^{c}}+\underbrace{I_{v}+\frac{5}{2} \phi_{m} I_{v}^{1 / 2}}_{\mu^{h}} .
$$

The contact contribution $\mu^{c}\left(I_{v}\right)$ is chosen similar to that reported in granular media, with $\mu_{1}=0.32$ (as observed here), $\mu_{2}=0.7$ and $I_{0}=0.005$ in agreement with available literature [10]. The hydrodynamic contribution $\mu^{h}\left(I_{v}\right)$ is designed to recover Einstein viscosity at low $\phi$ (see below) and gives the nonsaturating behavior at large $I_{v}$. This modeling fully captures experimental observation, over the whole range of viscous number $I_{v}$, regardless of particle size, material, and interstitial fluid (see solid lines in top Fig. 2).

A consistent model for the evolution of the volume fraction as a function of $I_{v}$ should have the asymptotic form $\phi_{m}-\phi \propto I_{v}^{1 / 2}$ at vanishing $I_{v}$, and stay positive for all values of $I_{v}$. The following function

$$
\phi\left(I_{v}\right)=\frac{\phi_{m}}{1+I_{v}^{1 / 2}},
$$

satisfactorily models the present measurements without any fitting parameter (see solid lines in bottom Fig. 2).

Recasting these latter equations to obtain $\phi$-dependent constitutive laws of dense suspensions then leads to

$$
\begin{gathered}
\eta_{s}(\phi)=1+\frac{5}{2} \phi\left(1-\frac{\phi}{\phi_{m}}\right)^{-1}+\mu^{c}(\phi)\left(\frac{\phi}{\phi_{m}-\phi}\right)^{2}, \\
\eta_{n}(\phi)=\left(\frac{\phi}{\phi_{m}-\phi}\right)^{2},
\end{gathered}
$$

with $\mu^{c}(\phi)=\mu_{1}+\left(\mu_{2}-\mu_{1}\right) /\left[1+I_{0} \phi^{2}\left(\phi_{m}-\phi\right)^{-2}\right]$. Again these constitutive laws fit the experimental data well, even for values very close to $\phi_{m}$ (Fig. 3). In this model, the effective shear viscosity $\eta_{s}(\phi)$ comprises three terms. The first two terms come from the hydrodynamic contribution and tend to Einstein viscosity $(1+5 \phi / 2)$ at $O(\phi)$. The third term is due to solid contacts and provides the leading divergence in $\left(\phi_{m}-\phi\right)^{-2}$. The effective normal viscosity $\eta_{n}(\phi)$ presents the same divergence and agrees well with proposed correlations $[15,17]$.

The present study provides an alternative viewpoint of dense suspension rheology. The granular paradigm is demonstrated to successfully describe the behavior of suspensions of hard spheres in a viscous fluid. An analytical model consistent with both frictional and hydrodynamic interactions is proposed. This can be easily generalized to three-dimensional complex flows [7] and applications in which the granular phase is subjected to gravitational forces. Another important result is the examination of the rheology close to the jamming transition. Using pressureimposed flows circumvents the divergences observed in $\phi$-imposed rheology at the jamming transition. Of fundamental importance is the finding of the critical (or maximum) volume fraction $\phi_{m}=0.585 \pm 0.002$ at the jamming point as well as the algebraic divergences of the effective viscosities in $\left(\phi_{m}-\phi\right)^{-2}$. The present study opens the path to future comparison with theoretical and numerical studies on the jamming transition of disordered particulate systems [18].

*francois.boyer@polytech.univ-mrs.fr

[1] A. Einstein, Ann. Phys. (Berlin) 322, 549 (1905). 
[2] J. J. Stickel and R. L. Powell, Annu. Rev. Fluid Mech. 37, 129 (2005).

[3] A. J. Liu and S. R. Nagel, Jamming and Rheology (Taylor \& Francis, London, 2001); Nature (London) 396, 21 (1998).

[4] P. Olsson and S. Teitel, Phys. Rev. Lett. 99, 178001 (2007).

[5] GDR MiDi, Eur. Phys. J. E 14, 341 (2004).

[6] F. da Cruz et al., Phys. Rev. E 72, 021309 (2005).

[7] P. Jop, Y. Forterre, and O. Pouliquen, Nature (London) 441, 727 (2006).

[8] Y. Forterre and O. Pouliquen, Annu. Rev. Fluid Mech. 40, 1 (2008).

[9] H. M. Jaeger, S. R. Nagel, and R. P. Behringer, Rev. Mod. Phys. 68, 1259 (1996).

[10] C. Cassar, M. Nicolas, and O. Pouliquen, Phys. Fluids 17,
103301 (2005).

[11] D. Prasad and H. K. Kytömaa, Int. J. Multiphase Flow 21, 775 (1995).

[12] T. Divoux and J.-C. Géminard, Phys. Rev. Lett. 99, 258301 (2007).

[13] J. A. Dijksman et al., Phys. Rev. E 82, 060301 (2010).

[14] A. N. Schofield and C.P. Wroth, Critical State Soil Mechanics (McGraw-Hill, New York, 1968).

[15] J. F. Morris and F. Boulay, J. Rheol. 43, 1213 (1999).

[16] A. Deboeuf et al., Phys. Rev. Lett. 102, 108301 (2009).

[17] P. Mills and P. Snabre, Eur. Phys. J. E 30, 309 (2009).

[18] T. K. Haxton, M. Schmiedeberg, and A. J. Liu, Phys. Rev. E 83, 031503 (2011). 